\title{
Prevalence and Short Term Outcome of Spontaneous Bacterial Peritonitis of Known Chronic Liver Disease Patients
}

Ismail $\mathrm{M}^{1}$, Rahman $\mathrm{MA}^{2}$

\begin{abstract}
Spontaneous bacterial peritonitis (SBP) is an infection of ascitic fluid occurring in the absence of a contiguous source of infection characterized by symptoms of fever, abdominal pain, rebound tenderness, encephalopathy. It may develope in hospitalized patients and mortality rate is significantly high. To determine the prevalence of SBP in chronic liver disease with ascites and to establish that SBP is the cause of higher mortality than non $S B P$, a prospective longitudinal study was carried out in patients attending in the inpatient Department of Gastroenterology of Bangladesh Institute of Research and Rehabilitation in Diabetes, Endocrine and Metabolic Disorder Hospital(BIRDEM), Bangabandhu Sheikh Mujib Medical University(BSMMU), Dhaka Medical College Hospital(DMCH), Dhaka from March 2010 to September 2010. Among the 60 patients the most common age group was the 46-55 years. In physical finding below average body build was found in 48 (80.0\%) cases. Malnutrition was found in 48(80.0\%) cases. Per abdominal finding liver was not palpable in 54 (90.0\%) cases. Shifting dullness was found in 59 (98.3\%) cases. Fluid thrill was detected in 57 (95.0\%) cases. It was found that SBP were developed in 11(18.3\%) cases and remaining 49(81.7\%) case were non SBP, which were higher than SBP. Organism of culture of ascitic fluid in SBP patients $(n=11)$ were E. coli and Pseudomoas spp found in 2(18.2\%) cases, the rest $6(54.5 \%)$ cases shows no growth. Among 11 SBP patients improvement occurred in 5(45.5\%) cases and the rest 6(54.5\%) cases died p value <.001. SBP is medical emergency, prompt management and prophylactic antibiotics are essential to reduce mortality.
\end{abstract}

Keywords: Spontaneous Bacterial Peritonitis, Chronic Liver Disease, Prevalence

\section{Introduction}

Spontaneous bacterial peritonitis (SBP) is an infection of ascitic fluid occurring in the absence of a contiguous source of infection (eg. Intestinal perforation, interabdominal abscess) characterized by symptoms of fever, abdominal pain, rebound tenderness, encephalopathy ${ }^{1}$.

1. Corresponding Author:

Mohammad Ismail

Assistant Professor, Department of Gastroenterology

Dhaka Medical College,Dhaka, Bangladesh

e-mail: drismailmd28@gmail.com

2. Mohammad Anisur Rahman

Professor \& Head of the Department

Gastrointestinal, Hapatobiliary and Pancreatic Disorders BIRDEM, Dhaka, Bangladesh
Between 10 and $30 \%$ of patients with cirrhosis develop $\mathrm{SBP}^{2}$, which carries hospital mortality rate ranging from 30 to $50 \%$. The risk of SBP recurrence is $70 \%$ at 1 st year ${ }^{4}$.

The presence of at least 250 polymorphonuclear cells per cubic millimeter of ascitic fluid is diagnostic of this condition. Aerobic gram-negative bacteria, primarily Escherichia coli, are the most common isolates, although the frequency of episodes caused by gram-positive bacteria has recently increased ${ }^{4}$.

Spontaneous bacterial peritonitis (SBP) is particularly frequent if the cirrhosis is severely decompensated. It is most characteristic infectious complication of cirrhosis. The occurrences of SBP are independent of the aetiology of liver diseases. The aim of the present study is to evaluate the prevalence and outcome of SBP patients with cirrhotic ascites.

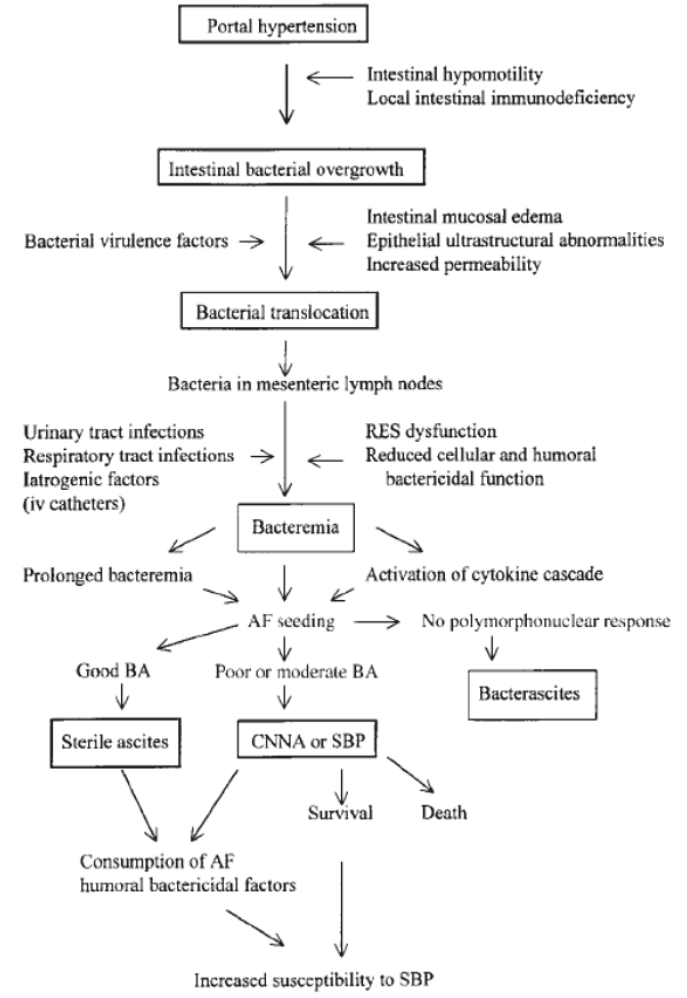

Figure 1. Mechanisms that may be involved in the pathogenesis of spontaneous bacterial peritonitis5. $\mathrm{AF}=$ ascitic fluid; $\mathrm{BA}=$ bacteri cidal activity; CNNA=culture-negative neutrocytic ascites; $\mathrm{RES}=$ reticuloendothelial system; $\mathrm{SBP}=$ spontaneous bacterial peritonitis 


\section{Materials and Methods}

Type of Study

This was a prospective longitudinal study.

Place of Study

This study was carried out in patients attending in the inpatient Department of Gastroenterology of BIRDEM Hospital, Bangabandhu Sheikh Mujib Medical University, Dhaka Medical College Hospital, Dhaka. This study was done from March 2010 to September 2010 for a period of 6 months. Study population were all ascitic patient admitted in defined period in the Department of Gastroenterology, BIRDEM Hospital, Bangabandhu Sheikh Mujib Medical University, Dhaka Medical College Hospital, Dhaka.

Sample size determination

Sample Size was determined by Prevalence of SBP in Bangladesh is not known. In India it is $37 \% 6$. Due to use of prophylactic and gut sterilizer (quinolone), reduces the incidence of SBP dramatically.

Sample size: $n=\frac{Z^{2} \times p q}{d^{2}}$

Here $\mathrm{z}=1.96, \mathrm{p}=0.37, \mathrm{q}=1-\mathrm{p}$ ) $\mathrm{d}$ (if degree of accuracy 0.05 ) from the formula, calculated sample size $=358(\mathrm{n})$, if population size 10,000 . In BIRDEM \& Other above mentioned hospital we have 4 cases per week and 96 cases per 6 Months $(\mathrm{N})$. In that case sample size would be

$$
n f=\frac{n}{1+\frac{n}{N}}=\frac{185}{1+\frac{185}{96}}=63
$$

So, a total number of 60 patients were taken as case of this study.

The sampling technique was purposive sampling method and consecutive hospital admission during define period. This purposive sampling was used as per inclusion and exclusion criteria.

Selection Criteria of Subjects

Inclusion Criteria

- Diagnosed case of chronic liver disease with ascites

- Patients not having any antibiotic within 2 weeks prior to hospital admission

Exclusion Criteria

Patients having following clinical condition were excluded from this study-

- Acutely ill patients.

- Patient who had taken any antibiotic within 2 weeks prior admission in ward

- Surgically treatable conditions (perforation of hollow viscous; appendicular lump; abscess).

- Patients or attendants unwilling to take part in the study

- Patients with congestive cardiac failure, nephrotic syndrome, peritoneal tuberculosis, abdominal malignancy.

Data Collection Procedure

Study procedure was followed under aseptic precaution. 20 $\mathrm{ml}$ of ascitic fluid was drawn by $20 \mathrm{cc}$ disposable syringe from right or left flank or midline of abdomen. $10 \mathrm{ml}$ ascitic fluid was taken in a heparinized tube to prevent clotting and examined within 1 hour for cell count. The remaining $10 \mathrm{ml}$ was inoculated in blood culture bottle containing tryptica soya broth $(100 \mathrm{ml})$ which was previously prepared.

Data was collected by using patient's information sheet which included clinical features, laboratory investigation that included routine ascitic fluid-tests, ascitic fluid culture, blood test-prothrombin time, serum billirubin, platelet count and serum creatinine, complete blood count.

Data were collected by investigator himself. Information was collected by taking medical history and clinical examination. Permission was taken from the concerned departments and informed written consent of each patient was taken in a consent form before collecting data.

Data analysis procedure

All data were recorded systematically in preformed data collection form and quantitative data was expressed as mean and standard deviation and qualitative data was expressed as frequency distribution and percentage. Statistical analysis was performed by using SPSS (Statistical Package for Social Sciences) for windows version 15 . Probability value $<0.05$ was considered as level of significance.

Ethical clearance

Prior to the commencement of this study, the thesis protocol was approved by the local ethical review committee of Diabetic Association of Bangladesh (BADAS). And informed written consent was taken from each patient.

\section{Results}

A total number of 60 patients of both sexes presented with ascites and admitted in the Department of Gastroenterology BIRDEM hospital, Bangabandhu Sheik Mujib Medical University, Dhaka Medical College Hospital, Dhaka were enrolled in this study.

Among the 60 patients the most common age group was the 46-55 years. The mean age of the study population was $49.78 \pm 15.22$ with a range of $16-80$ years. Among the study population the overall male and female ratio was 2.5: 1 . 
Etiology of hepatitis viruses among study population Hepatitis B, Hepatitis C and Non B-Non C were found in $45(75 \%)$ cases, $13(21.7 \%)$ cases and 2(3.3\%) cases respectively. Co-morbid conditions diabetes mellitus was found in $13(21.7 \%)$ cases and chronic renal failure was found in $3(5.0 \%)$ cases. Past history among the study population, jaundice was found in 53 (88.3\%) cases, abdominal swelling was found in 56 (93.3\%) cases, abdominal pain was found in 43(71.7\%) cases, disorientation was found in $49(81.7 \%)$ cases, blood vomiting was found in $25(41.7 \%)$ cases, bleeding per rectum was found in $16(26.7 \%)$ cases, black tarry stool was found in $43(71.7 \%)$ cases.
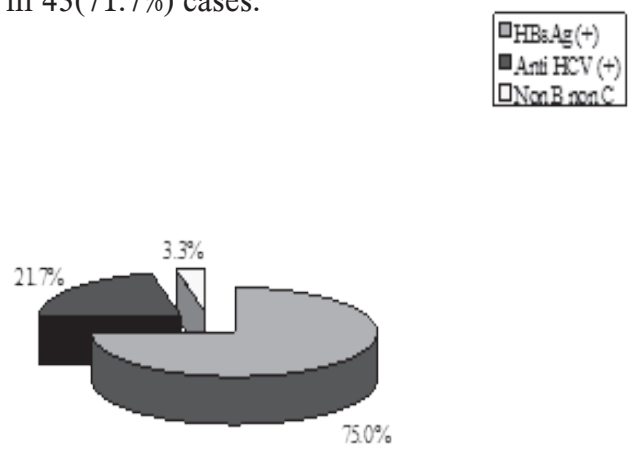

Figure 2. Pie chart of etiology of chronic liver disease among study population $(n=60)$

Patients were admitted in hospital with these clinical features. Fever was found among 47 (78.3\%) cases, abdominal pain was reported in 51(85.0\%) cases, abdominal tenderness was found in 46(76.7\%) cases, altered mentation was found in $40(66.7 \%)$ cases, and abdominal swelling was recorded in $41(68.3 \%)$ cases.

Table 1. Physical finding among the study population $(n=60)$

\begin{tabular}{lcc|}
\hline Physical findings & Frequency & Percentage (\%) \\
\hline Body build & 12 & 20.0 \\
Average & 48 & 80.0 \\
Below average & & \\
Nutrition & 48 & 80.0 \\
Malnutrition & 12 & 20.0 \\
Normal & & \\
Anaemia & 17 & 28.3 \\
Mild & 23 & 38.3 \\
Moderate & 20 & 33.3 \\
Severe & & \\
Stigma of CLD & 25 & 41.66 \\
Gynocomastia & 20 & 33.33 \\
Testicular atropy & 9 & 15.0 \\
Spider & 6 & 10.0 \\
Venous engorgement & 22 & 36.7 \\
Clubbing & 21 & 35.0 \\
Edema & 19 & 31.7 \\
Echymosis & 34 & 56.7 \\
Flapping tremor & & \\
\hline
\end{tabular}

MEDICINE 2015 Volume 27 Number 01
Physical finding among the study population was shown that below average body build was found in $48(80.0 \%)$ cases. Malnutrition was more common and was found in $48(80.0 \%)$ cases, moderate anaemia was seen in $23(38.3 \%)$ case and severe anaemia was seen in 20 (33.3\%).Stigma of CLD - gynocomastia was found in $25(41.66 \%)$ cases, testicular atrophy was found in $20(33.33 \%)$ cases, clubbing was found in $22(36.7 \%)$ cases. Edema was found in 21 (35.0\%) cases. Flapping tremor was found in 34 (56.7\%) cases. Spider nevae, venous engorgment, echymosis was also found .

Table 2. Per abdominal finding among the study population $(n=60)$

\begin{tabular}{lcc|}
\hline Per abdominal finding & Frequency & Percentage (\%) \\
\hline Abdominal swelling & 2 & 3.3 \\
Mild & 40 & 66.7 \\
Moderate & 18 & 30.0 \\
Tense & & \\
Liver & 6 & 10.0 \\
Palpable & 54 & 90.0 \\
Non palpable & 59 & 98.3 \\
Shifting dullness & 57 & 95.0 \\
Fluid thrill &
\end{tabular}

Regarding per abdominal finding moderate abdominal swelling was seen $40(66.7 \%)$ cases and tense abdominal swelling was seen $18(30.0 \%)$ cases. Mostly liver was non palpable in $54(90.0 \%)$ cases. Shifting dullness was found in $59(98.3 \%)$ cases. Fluid thrill was detected in $57(95.0 \%)$ cases.

Table 3. Laboratory investigation in SBP and non SBP patients $(n=60)$

\begin{tabular}{lll|}
\hline Laboratory investigation & SBP $(\mathrm{n}=11)$ & Non SBP $(\mathrm{n}=49)$ \\
\hline S. billirubin $(\mathrm{mg} / \mathrm{dl})$ & $4.18 \pm 1.69$ & $4.32 \pm 3.76$ \\
S. albumin $(\mathrm{g} / \mathrm{l})$ & $2.48 \pm 0.47$ & $2.59 \pm 0.68$ \\
ALT(iu/l) & $89.64 \pm 83.90$ & $87.65 \pm 79.73$ \\
Prothrombin time(sec) & $26.31 \pm 6.32$ & $21.48 \pm 5.30$ \\
Plateletcount $/ \mathrm{mm} 3)$ & $21178.98 \pm 4983.27$ & $32854.55 \pm 26754.3$ \\
S. creatinine $(\mathrm{mg} / \mathrm{dl})$ & $1.51 \pm 0.23$ & $1.22 \pm 0.34$ \\
Hb $(\mathrm{gm} / \mathrm{dl})$ & $8.24+.10$ & $9.04 \pm .51$ \\
TC $(/ \mathrm{mm} 3)$ & $13915.4 \pm 8810.85$ & $12794.5 \pm 9739.05$ \\
Neutrophil $\%$ & $77.36 \pm 8.83$ & $75.46 \pm 14.75$ \\
Lymphocyte\% & $16.27 \pm 6.90$ & $19.44 \pm 14.23$ \\
Monocyte $\%$ & $3.36 \pm 1.21$ & $3.04 \pm 2.53$ \\
Eosinophil\% & $2.73 \pm 2.15$ & $1.97 \pm 1.40$ \\
\hline
\end{tabular}

Data was expressed as Mean \pm SD.

Table 4. Prevalence of SBP among the study population $(\mathrm{n}=60)$

\begin{tabular}{cccc}
\hline Total study subjects & SBP & Percentage of SBP & 95\% CI \\
\hline 60 & 11 & $18.3 \%$ & $8.54-28.12$ \\
\hline
\end{tabular}

CI- Confidence interval 
Among 60 ascitic patients SBP were developed in $11(18.3 \%)$ cases and remaining $49(81.7 \%)$ case were non SBP, which were higher than SBP.

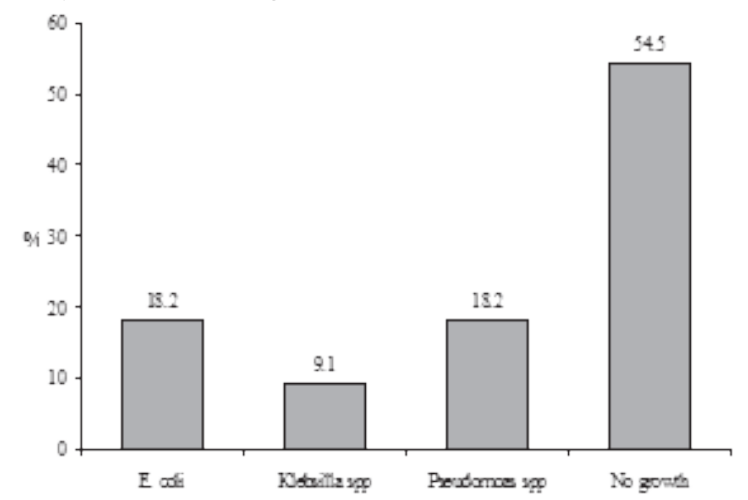

Figure 3. Organism of culture of ascitic fluid in SBP patients $(n=11)$

E. coli and Pseudomonas spp were found in 2(18.2\%) cases in each. Only 1(9.1\%) Klebsilla spp was found among the study population. The rest $6(54.5 \%)$ cases showed no growth.

Table 5. Mortality in SBP and non SBP patients $(n=60)$

\begin{tabular}{cccc|}
\hline Mortality & SBP $(\mathrm{n}=11)$ & Non SBP $(\mathrm{n}=49)$ & $\mathrm{p}$ value* \\
\hline Dead & $6(54.5)$ & $4(8.2)$ & \\
Improved & $5(45.5)$ & $45(91.8)$ & .001 \\
Total & $11(100.0)$ & $49(100.0)$ & \\
\hline
\end{tabular}

*Fisher's Exact test was done to measure the level of significance.

Among 11 SBP patients improvement occurred in $5(45.5 \%)$ cases and the rest $6(54.5 \%)$ cases died.

\section{Discussion}

Spontaneous bacterial peritonitis (SBP) is a lethal complication of chronic liver disease ${ }^{1}$ and is diagnosed when the ascitic fluid culture shows growth of a monomicrobial Gram negative organism, ascitic fluid neutrophils count $\geq 250$ cells $/ \mathrm{mm}^{3}$ and without evidence of surgically treatable intra abdominal conditions ${ }^{1}$.

The prevalence of SBP in the community ranges from 5\% to $10 \%{ }^{7}$ and in hospitalized patient from $10 \%$ to $30 \%{ }^{8}$. Even with intensive treatment, hospital mortality is still between $10 \%$ and $30 \%{ }^{9}$. Factors associated with poor outcome include hepatic encephalopathy, high serum bilirubin, gastrointestinal bleeding and renal failure ${ }^{8}$ SBP is due to translocation of bacteria from gut to peritonium and related to low protein levels and impaired opsonic activity in ascitic fluid1.

A total number of 60 patients of both sexes presented with ascites and admitted in the Department of Gastroenterology BIRDEM Hospital, Bangabandhu Sheik Mujib Medical University and Dhaka Medical College
Hospital, Dhaka were enrolled in the study. Among the 60 patients the most common age group was the $46-55$ years which was $25.0 \%$ followed by $56-65$ years, $36-45$ years, $>65$ years, 26-35 years and less than or equal to 25 years which were $23.3 \%, 18.3 \%, 13.3 \%, 11.7 \%$ and $8.3 \%$ respectively. Similar result was found and reported that the higher age group were more vulnearable to SBP, due to more chance of infection to those patients ${ }^{10}$. Another study also showed that middle age group was the most common age group ${ }^{11}$.

Among the study population male was predominant. The overall male and female ratio was 2.5: 1 . Similar result was reported and mentioned that $69.6 \%$ were males and $30.4 \%$ were female ${ }^{11}$.

In this study Hepatitis B, Hepatitis C and Non B-Non C was $45(75 \%), 13(21.7 \%)$ and $2(3.3 \%)$ respectively and a similar result also were reported ${ }^{11}$.

In this present study co-morbid condition DM was present in $13(21.7 \%)$ and $\mathrm{CRF}$ was $3(5.0 \%)$. In a study ${ }^{4}$ the mortality rate was $100 \%$ when associated with progressive renal impairment, $31 \%$ when associated with steady renal impairment, and only $7 \%$ in those without renal impairment. Filik and $\mathrm{Unal}^{11}$ were reported a similar result.

According to past history it was found that jaundice was present in $53(88.3 \%)$ cases; abdominal swelling was 56 (93.3\%); abdominal pain was $43(71.7 \%)$; disorientation was 49(81.7\%). Similar result was reported ${ }^{9}$ and added that the clinical manifestations of SBP are subtle. In this study it was found that blood vomiting was $25(41.7 \%)$; bleeding per rectum $16(26.7 \%)$; black tarry stool was $43(71.7 \%)$. Similar result was reported in another study ${ }^{11}$.

In this study presenting chief complains were seen as fever was found among $47(78.3 \%)$ cases, similar result was reported and mentioned that fever caused by SBP is differentiated from that of alcoholic hepatitis ${ }^{4}$. Abdominal pain was reported $51(85.0 \%)$ cases; abdominal tenderness was present in $46(76.7 \%)$ cases. Similar result was reported that abdominal pain can be continuous and is different from tense ascites ${ }^{2,11}$. Altered mentation was 40(66.7\%); abdominal swelling was $41(68.3 \%)$; nausea or vomiting was 23( $38.3 \%$ ); blood vomiting was $24(40.0 \%)$; bleeding per rectum was $10(16.7 \%)$; black tarry stool was $24(40.0 \%)$; respiratory infection was $19(31.7 \%)$, shortness of breathing was $29(48.3 \%)$; only $3(5.0 \%)$ was asymptomatic. From another study, similar result were shown and added that all spontaneous bacterial peritonitis were symptomatic ${ }^{11}$.

According to physical finding, maximum body build $48(80.0 \%)$ was below average; most of the respondents 48 $(80.0 \%)$ were malnourished and all the cases anaemia common. In stigma of chronic liver disease, gynocomastia was present in $25(41.66 \%)$ cases; testicular atropy was $20(33.33 \%)$; spider nevae was $9(15.0 \%)$; venous engorgment was $6(10.0 \%)$; clubbing was $22(36.7 \%)$; edema was $21(35.0 \%)$; echymosis was 19 ( $31.7 \%)$; flapping tremor was $34(56.7 \%)$. The most frequently encountered 
symptoms and signs are fever $69 \%$, abdominal pain $59 \%$, signs of hepatic encephalopathy, abdominal tenderness, ileus, shock and hypothermia ${ }^{12}$.

In per abdominal finding abdominal swelling was more in moderate and tense abdomen than mild and most of the cases $54(90 \%)$ liver was non palpable. Shifting dullness was found 59(98.3\%) cases. Fluid thrill was 57(95.0\%). From a study $^{13}$ it is found that at physical examination, patients with ascites and SBP do not have a rigid abdomen because the ascitic fluid in great amounts prevents the contraction between the peritoneal membranes.

Laboratory investigation was done in both SBP and nonSBP, there was no significant difference between two groups. It was mentioned that a serum total bilirubin level $(\mathrm{mg} / \mathrm{dl})$ of $2.5 \mathrm{mg} / \mathrm{dL}$ is an independent predictive factor of $\mathrm{SBP}^{14}$. A direct correlation between total protein level, complement components, and opsonic activity explains that an asitic fluid total protein level of $<1 \mathrm{~g} / \mathrm{di}$ is a risk factor for the development of asitic fluid infection ${ }^{15}$. It was reported that patients with cirrhosis have coagulation disturbances which was consistant with this study ${ }^{14}$. Similar laboratory findings were reported and mentioned that the factors associated with poor outcome include hepatic encephalopathy, high levels of serum bilirubin, and gastrointestinal bleeding ${ }^{8}$.

Ascitic fluid was cultured of SBP patients and were found E. coli and Pseudomoas spp in 2(18.2\%) cases in each. Only $1(9.1 \%)$ Klebsilla spp was found among the study population. The rest $6(54.5 \%)$ cases shows no growth. Organisms that cause SBP is Gram negative as because of its predominance in gut flora. similar result was found that an increased frequency of the SBP episodes produced by Gram negative bacteria has been ascertained ${ }^{12}$ and also reported that more than $60 \%$ of SBP episodes are caused by Gram negative enteric bacteria which is consistant with this study and added that Escherichia coli and Klebsiella pneumoniae are the organisms isolated most frequently which was similar to this study ${ }^{14}$.

Limitations of the Study

There are some limitations in this study. Some are mentioned below:

1. Sample size was small

2. It was a non randomized sampling method

3. The study and follow up period was short in comparison to other series.

In conclusion, SBP is common in chronic liver disease with ascites. Clinical feature and biochemical marker are almost same in SBP and non-SBP. The most common bacteria that isolated from SBP patients are the E coli, Klebsilla species and Pseudomonas species. After development of SBP mortality is high for that SBP is a medical emergency, prompt management and prophylactic antibiotics is essential to reduce mortality.

\section{References}

1. Schiano Thmas D,Bodenhemer Henry C. Complications of Chronic liver Disease.In: Friedman Scott L,Macqid Kenneth R,Grandell James H(eds) Current diagnosis \& treatment of Gastroenterology $(2 \mathrm{nd}$ ed);2002;43:639-663.

2. Rimola A, Garcia-Tsao G, Nacasa M et al. Diagnosis, treatment and prophylaxis of spontaneous bacterial peritonitis: a consensus document. J Hepatol 2000;32:142 - 3

3. Gines P, Rimola A, Planas R e t al. Norfloxacin prevents spontaneous bacterial peritonitis recurrence in cirrhosis: results of a double-blind, placebo-controlled trial. Hepatology 1990;12:716 - 24

4. Gines P, Cardenas A, Arroyo V et al. Management of cirrhosis and ascites . N Engl J Med 2004;350 : 1646 4

5. Wiest R, Garcia-Tsao G. Bacterial translocation in cirrhosis. Hepatology 2005; 41: 422- 433

6. Prevalence of spontaneous Bacterial Peritonitis J. Assoc. Physicians India. 1992;40:236-8.

7. Kline MM, McCallum RW, Guth PH. The clinical value of ascitic fluid culture and leukocyte count studies in alcoholic cirrhosis. Gastroenterology 1976; 70:408-12

8. Parsi MA, Atreja A, Zein NN. Spontaneous bacterial peritonitis: Recent data on incidence and treatment. Cleveland Clinic Journal Of Medicine 2004;71:569-576

9. Garcia-Tsao G. Current management of the complications of cirrhosis and portal hypertension: variceal hemorrhage, ascites, and spontaneous bacterial peritonitis. Gastroenterology 2001;120:726-748

10. Syed VA, Ansari JA, Karki P, Regmi M, Khanal B. Spontaneous bacterial peritonitis (SBP) in cirrhotic ascites: A prospective study in atertiary care hospital, Nepal. Kathmandu University Medical Journal 2007;51:48-59

11. Filik L, Unal S. Clinical and laboratory features of spontaneous bacterial peritonitis. East African Medical Journal. 2004:819:474-479

12. Crruntu FA, Benea L. Spontaneous Bacterial Peritonitis: Pathogenesis, Diagnosis, Treatment. J Gastrointest Liver Dis 2006;151:51-56

13. Evans LT, Kim WR, Poterucha JJ, Kamath PS. Spontaneous bacterial peritonitis in asymptomatic outpatients with cirrhotic ascites. Hepatology 2003;37: $897-901$

14. Such J, Runyon BA. Spontaneous bacterial peritonitis. Clin Infect Dis 1998;27:669-674

15. Runyon BA. Patients with deficient ascitic fluid opsonic activity are predisposed to spontaneous bacterial peritonitis. Hepatology 1988;8:632-5 